\title{
Ku proteins interact with activator protein-2 transcription factors and contribute to ERBB2 overexpression in breast cancer cell
} lines

\author{
Grégory Nolens ${ }^{1}$, Jean-Christophe Pignon1, Benjamin Koopmansch¹, Benaïssa Elmoualij2, \\ Willy Zorzi ${ }^{2}$, Edwin De Pauw ${ }^{3}$ and Rosita Winkler ${ }^{1}$
}

\author{
1'Laboratory of Molecular Oncology, GIGA Cancer, University of Liège, B34, avenue de I'hopital, Liege, 4000, Belgium \\ 2Department of Human Histology-CRPP, University of Liège, B36, avenue de l'hopital, Liege, 4000, Belgium \\ ${ }^{3}$ Laboratory of Mass Spectrometry; CART, GIGA, University of Liège, B6, avenue de la chimie, Liege, 4000, Belgium \\ Corresponding author: Grégory Nolens, gnolens@gmail.com
}

Received: 26 Apr 2009 Revisions requested: 22 May 2009 Revisions received: 7 Oct 2009 Accepted: 11 Nov 2009 Published: 11 Nov 2009

Breast Cancer Research 2009, 11:R83 (doi:10.1186/bcr2450)

This article is online at: http://breast-cancer-research.com/content/11/6/R83

(c) 2009 Nolens et al.; licensee BioMed Central Ltd.

This is an open access article distributed under the terms of the Creative Commons Attribution License (http://creativecommons.org/licenses/by/2.0), which permits unrestricted use, distribution, and reproduction in any medium, provided the original work is properly cited.

\begin{abstract}
Introduction Activator protein-2 (AP-2) $\alpha$ and AP- $2 \gamma$ transcription factors contribute to ERBB2 gene overexpression in breast cancer. In order to understand the mechanism by which the ERBB2 gene is overexpressed we searched for novel AP-2 interacting factors that contribute to its activity.

Methods $\mathrm{Ku}$ proteins were identified as AP-2 $\alpha$ interacting proteins by glutathione serine transferase (GST)-pull down followed by mass spectrometry. Transfection of the cells with siRNA, expression vectors and reporter vectors as well as chromatin immunoprecipitation (ChIP) assay were used to ascertain the implication of Ku proteins on ERBB2 expression.

Results Nuclear proteins from BT-474 cells overexpressing AP$2 \alpha$ and AP- $2 \gamma$ were incubated with GST-AP2 or GST coated beads. Among the proteins retained specifically on GST-AP2 coated beads Ku70 and Ku80 proteins were identified by mass spectrometry. The contribution of Ku proteins to ERBB2 gene expression in BT-474 and SKBR3 cell lines was investigated by downregulating Ku proteins through the use of specific siRNAs.
\end{abstract}

Depletion of $\mathrm{Ku}$ proteins led to downregulation of ERBB2 mRNA and protein levels. Furthermore, reduction of Ku80 in HCT116 cell line decreased the AP- $2 \alpha$ activity on a reporter vector containing an AP-2 binding site linked to the ERBB2 core promoter, and transfection of Ku80 increased the activity of AP$2 \alpha$ on this promoter. Ku siRNAs also inhibited the activity of this reporter vector in BT-474 and SKBR3 cell lines and the activity of the ERBB2 promoter was further reduced by combining $\mathrm{Ku}$ siRNAs with AP- $2 \alpha$ and AP- $2 \gamma$ siRNAs. ChIP experiments with chromatin extracted from wild type or AP- $2 \alpha$ and AP- $2 \gamma$ or Ku70 siRNA transfected BT-474 cells demonstrated Ku70 recruitment to the $E R B B 2$ proximal promoter in association with AP- $2 \alpha$ and AP- $2 \gamma$. Moreover, Ku70 siRNA like AP-2 siRNAs, greatly reduced Polll recruitment to the ERBB2 proximal promoter.

Conclusions $\mathrm{Ku}$ proteins in interaction with AP-2 ( $\alpha$ and $\gamma$ ) contribute to increased ERBB2 mRNA and protein levels in breast cancer cells.

\section{Introduction}

Breast cancer is the most common cancer in women in Europe [1]. Accumulation of different molecular alterations characterizes this complex disease. Five major breast cancer subgroups have been distinguished according to gene expression signatures $[2,3]$. One of these subgroups is characterized by ERBB2/Her2 gene amplification and overexpression. This alteration is present in about $20 \%$ of breast cancers and was found to be predictive of poor prognosis before the development of ERBB2 targeted drugs [4-6].

The ERBB2 gene encodes for $\mathrm{p} 185^{-\mathrm{erbB}}$, which is a transmembrane protein with intrinsic tyrosine kinase activity belonging to the EGF receptor (EGFR) family. No growth factor 
recognizing specifically $E R B B 2$ with high affinity has been identified. Consequently, p185-erbB2 is assumed to be activated by hetero-dimerization with another ligand-activated member of the EGFR family [6].

The high levels of p185-erbB2 measured in breast cancer cells result from gene amplification and increased transcription rates $[7,8]$. In order to investigate the biology of these specific breast cancers, we chose to study the deregulation of $E R B B 2$ gene expression. Analyses of the ERBB2 promoter have led to the identification of several regulatory sequences through which the gene is overexpressed. AP-2, Ets and YB-1 transcription factor families bind to some of these regulatory regions and have been shown to play a role in $E R B B 2$ overexpression. Ets family transcription factors contribute to $E R B B 2$ overexpression by binding to the proximal promoter [9]. YB-1 factors act through binding sites located 815 to $1129 \mathrm{bp}$ upstream the main transcription initiation site [10], whereas AP-2 binding sequences (AP2BS) have been identified in the proximal [11-13] and distal [14] regions of the promoter.

The AP-2 transcription factor family contains five members: AP- $2 \alpha, \beta, \gamma, \delta$ and $\varepsilon$. All have a similar $50 \mathrm{kDa}$ apparent molecular mass and are able to form homo- and hetero-dimers. They bind specific DNA sequences, AP2BS, through their conserved helix-span-helix DNA binding domain.

The involvement of AP- $2 \alpha$ and AP- $2 \gamma$ factors in ERBB2 overexpression has been described in several breast cancer cell lines $[11-13,15]$. Besides the ERBB2 gene, AP-2 factors control the expression of several target genes implicated in the control of cell growth, differentiation and carcinogenesis [16].

AP-2 factors control transcription in association with transcriptional cofactors [17]. Among them, PC4, PARP [18], CITED2, CITED-4 and CBP/p300 [19], as well as YY1 [20], have been shown to interact with and to contribute to AP-2 transcriptional activity. In our own research, we have observed a good correlation between p185-erbB2, AP-2 $\alpha$ and YY1 expression levels in primary breast tumor samples [21]. Besides their role in transcription, cofactors are also important for the protection of AP-2 against proteasomal degradation [22].

In order to improve the current understanding of AP-2 ( $\alpha$ and $\gamma$ ) activity, we sought here to identify further AP- $2 \alpha$ interacting factors contributing to ERBB2 gene overexpression in breast cancer cells. We used a proteomic approach to isolate proteins interacting with this transcription factor in a BT-474 breast cancer cell line. Ku70 and Ku80 were identified by mass spectrometry among the AP- $2 \alpha$ interacting proteins.

Ku 70 and Ku80 hetero-dimers are mostly known for their role, in association with DNA-PK, in the repair of DNA double strand breaks. However, it has been shown that Ku70 and Ku80 are involved in transcription regulation either by binding directly to DNA or through interaction with transcription factors [23]. Ku factors might also play a role in cancer [24].

We show that siRNAs targeting $\mathrm{Ku}$ mRNAs downregulate ERBB2 mRNA and protein levels. The use of reporter vectors containing the $E R B B 2$ proximal promoter demonstrated that $\mathrm{Ku} 70$ and Ku80 proteins are involved in ERBB2 transcription regulation. Moreover, we show by ChIP assays that Ku70 protein is recruited to the $E R B B 2$ gene promoter and its absence decreases AP- $2 \alpha$ and AP- $2 \gamma$ recruitment. Furthermore, Ku70 recruitment is dependent on the expression of AP- $2 \alpha$ and AP$2 \gamma$. These results contribute to a better understanding of the mechanism by which AP-2 factors upregulate ERBB2 gene expression in breast cancer cells.

\section{Materials and methods Cell lines}

All the human cell lines (BT-474, ZR-75.1, MDA-MB-231, MCF-7 and SK-BR3, HepG2) were purchased from the American Tissue Culture Collection (Manassas, VA, USA). HCT116 and the derived 70/32 (Ku80+/-) cell lines were gifts from Dr. EA Hendrickson [25]. All the cells were cultured in the recommended media supplemented with 10\% (v/v) fetal bovine serum, $2 \mathrm{mM}$ glutamine and $100 \mu \mathrm{g} / \mathrm{ml}$ penicillin/streptomycin (Lonza, Basle, Switzerland).

\section{Antibodies}

Mouse anti-AP-2 $\alpha$ (3B5) [20], rabbit anti-AP-2 $\alpha$ (C-18) [20], mouse AP- $2 \gamma$ (6E4/4) [21], goat anti-Ku70 (C-19) [26], goat anti-Ku80 (C-20) [26], mouse anti-Ku80 (B-1) [27], and control mouse, goat and rabbit $\lg G$ antibodies were purchased from Santa Cruz Biotechnology (Santa Cruz, CA, USA. Anti RNA Polymerase II (clone CTD4H8) and anti- $\beta$-actin (mAbcam 8226) antibodies were obtained from Abcam (Cambridge, UK).

\section{Plasmids and constructs}

The pGEX2T-GST-AP2 and -GST vectors were gifts from Dr. Kannan [28]. The p86-AP2BS-Luc and p86-AP2BS mut-Luc plasmid reporter vectors (pGL3 basic reporter vector, Promega, Madison, WI, USA) have been previously described by Vernimmen et al [13]. The SV40-Luc control vector (pGL3 control vector) was purchased from Promega. The AP- $2 \alpha$ and the corresponding control expression vectors [29] have been described previously [20]. Ku80 expression vector was a gift from Dr. Chen [30].

\section{GST-pull-down}

GST-fusion proteins were expressed and purified according to the procedures provided by Amersham Bioscience (Buckinghamshire, UK). Their purification and the pull-down assay were carried out by using the MagneGST ${ }^{\mathrm{TM}}$ Pull-Down System (Promega, Madison, WI, USA). The manipulation (incubation and washing) of the beads was automated by the KingFisher robot (Thermo Fisher Scientific, Waltham, MA, USA). Wash- 
ing buffer was phosphate buffer saline (PBS)/0,01\% Tween. Procedure: $15 \mu \mathrm{l}$ of MagneGST beads were washed twice with the washing buffer. Beads were incubated with $50 \mu \mathrm{l}$ of sonicated E. coli $(\mathrm{BI} 21)$ transformed with plasmids encoding GST or GST-AP2alpha fusion protein in $250 \mu$ of PBS for 30 $\mathrm{min}$ at room temperature (RT). After washing twice with $400 \mu \mathrm{l}$ of washing buffer, beads bound with GST or GST-AP2 were incubated for 50 minutes with $100 \mu \mathrm{g}(35 \mu \mathrm{l})$ of nuclear proteins extracted from BT474 at RT in $180 \mu \mathrm{l}$ of PBS. After washing the beads three times with $400 \mu \mathrm{l}$, the interacting proteins were finally eluted by $8 \mathrm{M}$ urea for $2 \mathrm{D}$ gel electrophoresis, or with Laemmli Buffer (2\% SDS, 10\% glycerol, 5\% 2mercaptoethanol, $0.125 \mathrm{M}$ Tris $\mathrm{HCl} \mathrm{pH} \mathrm{6.8)} \mathrm{for} \mathrm{western} \mathrm{blot-}$ ting.

DNase I treatment - GST-AP2 beads incubated with the nuclear protein extracts were washed twice with PBS and suspended in DNase I buffer ( $40 \mathrm{mM}$ Tris $\mathrm{HCl}, 10 \mathrm{mM} \mathrm{NaCl}, 6$ $\mathrm{mM} \mathrm{MgCl} 2,1 \mathrm{mMCaCl} 2, \mathrm{pH} 7.9)$. The suspension was incubated with increasing concentrations of DNase I (Roche, Basel, Switzerland) for one hour at $37^{\circ} \mathrm{C}$. The suspension was washed three times with PBS, resuspended in Laemmli buffer and the bound proteins were eluted by vortexing during 10 min. Ku70, Ku80 and AP-2 were revealed by western blotting. DNA quantity was estimated by the picoGreen assay (Invitrogen, Carlsbad, CA, USA).

Two-dimensional gel electrophoresis and mass spectrometry

These techniques were performed as described previously [31], except that the proteins were directly loaded onto IPG strips (non linear IPG strip pH 4-10; Amersham, GE Europe (Buckinghamshire, UK). Liquid chromatography was carried out in an UltiMate ${ }^{\mathrm{TM}}$ pump/detection module, FAMOS $^{\mathrm{TM}}$ micro autosampler, Switchos ${ }^{\mathrm{TM}}$ micro switching module (LC Packings, Dionex, Sunnyvale, CA, USA). The mass analysis was carried out in an ion trap Esquire HCT (Bruker Daltonics, Bremen, Germany) mass spectrometer. The database search was performed using a Mascot local server (Matrix Science, London, UK).

\section{Immunoblotting}

Proteins were separated on an SDS-PAGE (10\%) and transferred to a PVDF membrane (Millipore, Billerica, MA, USA). Primary antibodies were used at a 1:1000 dilution. Secondary antibodies coupled with peroxydase (DAKO, Glostrup, Denmark) at a 1:4000 dilution were detected using the ECL system (Thermo Fisher Scientific).

Immunoprecipitation was carried out using Dynabeads Protein G (Invitrogen), according to the manufacturer's recommended protocol, using acetate sodium buffer for antibodies binding. Anti-AP-2 $\alpha$ (C-18), Ku70 (C-19), Ku86 (C-20) antibodies and control antibody were used.
Transient transfection assays of reporter vectors HCT116, 70/32 (HCT116 Ku80 +/-), BT-474 and SKBR3 cells were transfected using FuGENE HD reagent (Roche Applied Science). The cells $\left(3 \times 10^{5}\right)$ were plated onto $24 \mathrm{~mm}$ tissue culture dishes, treated with FuGENE HD/DNA (ratio of $3: 1)$ and incubated for $40 \mathrm{~h}$ in complete medium. Cells were then harvested. Lysis and enzymatic activity measures were carried out using the Luciferase Reporter Gene Assay kit (Roche Applied Science). Enzymatic activity was measured in a Wallac Victor ${ }^{\mathrm{TM}}$ luminometer (PerkinElmer, Waltham, MA, USA). The data were normalized to total protein content.

Transient siRNA transfection

siRNAs were transfected at a $30 \mathrm{nM}$ final concentration using the Calcium Phosphate precipitation technique [32]. Cells were transfected twice at $48 \mathrm{~h}$ intervals. As a control, cells were transfected with the negative control siRNA OR-0030neg05 (Eurogentec, Seraing, Belgium). The AP-2 siRNAs used were as previously described [14]. Other siRNAs were: Ku70-1, 5-GUGUGUACAUCAGUAAGAU; Ku70-2, 5-CAGGCAUCUUCCUUGACUU; Ku80-1, 5-GAAGAGGCAUAUUGAAAUA; Ku80-2, 5-CUCCAUUCCUGGUAUAGAA.

\section{Reverse Transcription-Polymerase Chain Reaction (RT- PCR)}

Total RNA was extracted after 90 hours of siRNA transfection treatment, using the High Pure RNA Isolation kit (Roche Applied Sciences). RNA quantification was carried out on a Nano Drop 1000 (Thermo Fisher Scientific). Reverse transcription was performed on $1 \mu \mathrm{g}$ of total extracted RNA. Real time PCR analysis was performed on an ABI Prism 5700 apparatus (Applied Biosystems, Foster City, CA, USA) using the standard protocol. All the results were reported to the $\beta-2$ microglobulin mRNA quantity. The primers used were as previously described [21], except for Ku70: 5-AGAAGCAAACCGCCTGTA and 5-CAAGCCTCCTCCAATAAAGC; and Ku80: 5-TGCAGCAAGAGATGATGAGG and 5-GAAAGGCAGCTGCACATACA.

\section{Chromatin Immunoprecipitation (ChIP)}

Chromatin Immunoprecipitation was carried out using Dynabeads Protein G (Invitrogen). The previously described protocol [33] was adapted for higher chromatin quantities. On average for each immunoprecipitation reaction, $30 \mu \mathrm{g}$ of chromatin DNA was precipitated with $4 \mu \mathrm{g}$ of antibody. Rabbit antiAP-2 $\alpha$ (C-18), goat anti-Ku70 (C-19) and mouse anti-Ku80 (C-20) were used. Immunoprecipitation with pre-immune sera from the same animal species (lg) or mock immunoprecipitation (NoAb) served as negative controls. Recovered DNA was quantified by real time PCR or by end-point PCR followed by agarose gel electrophoresis and the results were compared to known quantities of chromatin. The gene-specific primer sequences were: -6900 primers: 5-GCAGTAGCAAGCATCGAGTT and 5-TGGATCATCACAAAGGTTTTCA (-6981 bp to -6780 bp); -500-bp ERBB2 primers, 5-GACTGTCTCCTC- 
CCAAATTT and 5-CTTAAACTTTCCTGGGGAGC (fragment -575 to -349 bp); -100-bp ERBB2 primers, 5-GCGAAGAGAGGGAGAAAGTG and 5-GGGGAATCTCAGCTTCACAA; GCK primers, 5-GGTAGAGCAGATCCTGGCAG AG and 5-TGAGCCTTCTGGGGTGGAGCGCA. Dilutions of known quantities of input DNA were used to quantify the PCR products.

\section{Results}

\section{Identification of novel AP-2 $\alpha$ interacting proteins}

Our goal was to identify novel proteins interacting with AP-2, which contribute to the factor's transcriptional activity. Nuclear protein extracts from BT-474 breast cancer cells were incubated with a Glutathione-Serine-Transferase/AP-2 $\alpha$ (GSTAP2) hybrid protein, linked to glutathione (GSH) coated magnetic beads. Beads coated with expressed GST alone were used as a negative control. After washes, the proteins bound to GST-AP2 or GST coated beads were eluted and resolved on two-dimensional gel electrophoresis. Two proteins migrat- ing with an apparent molecular mass comprised between 70 and $100 \mathrm{kDa}$ were repeatedly detected among the proteins eluted exclusively from the GST-AP2 coated beads (Figure $1 \mathrm{~A})$. The corresponding spots were cut out of the gel, the proteins digested, and the resulting peptides analyzed by LC-MS/ MS. The proteins were identified as Ku70 and Ku80 [see Additional data file 1]. Most of the other spots on the 2D-gel recovered from the GST-AP2 pull down, were identified as GST or AP- $2 \alpha$ fragments.

The presence of Ku proteins among the proteins eluted from the GST-AP2 beads was confirmed by immunoblotting with Ku specific antibodies (Figure 1B). Moreover, the interaction between AP-2 and Ku proteins was controlled by co-immunoprecipitation, using AP-2, Ku70 and Ku80 specific antibodies (Figure $1 \mathrm{C}$ ). To verify that the binding of Ku proteins to AP2 is not due to the DNA contaminating the protein extracts [34], the mixture of GST-AP2 beads and nuclear proteins were incubated with increasing concentrations of DNase I. The

Figure 1

(a)

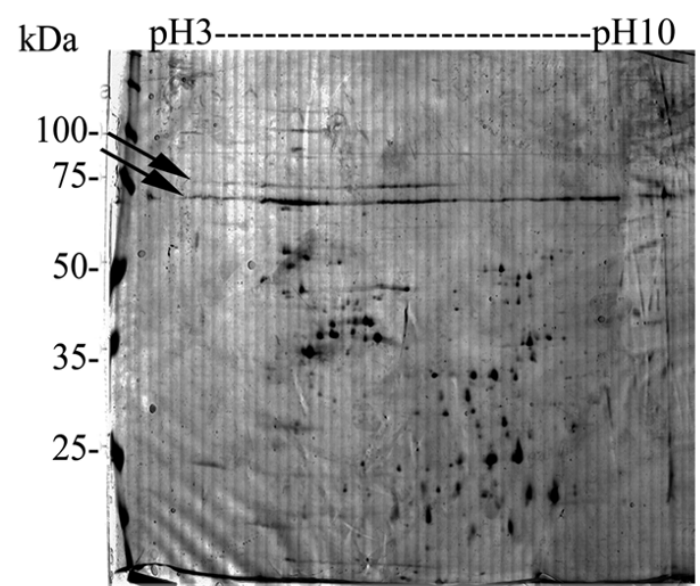

\section{GST}

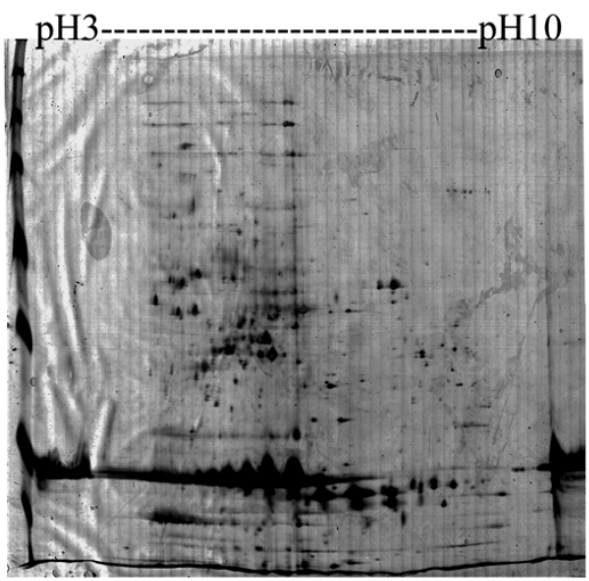

(b)

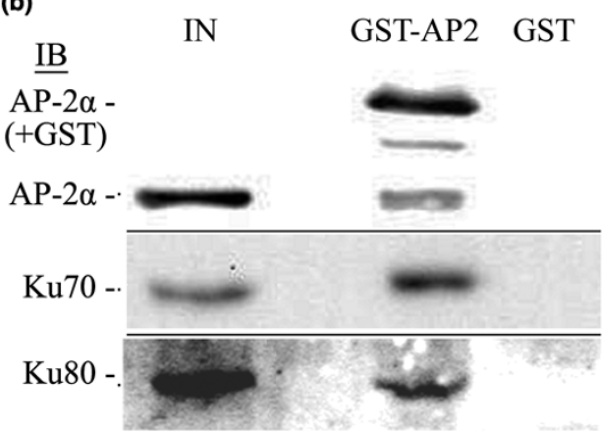

(c)

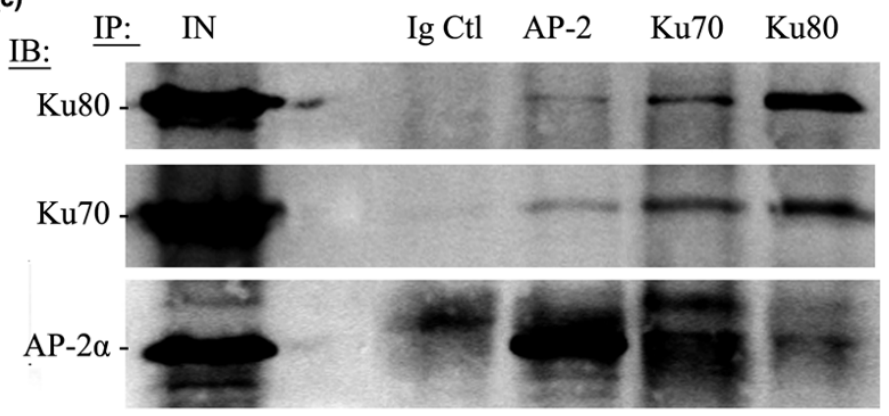

Identification of the Ku proteins interacting with AP-2 $\alpha$. (a) Two-dimensional gel electrophoresis of nuclear proteins eluted from GST AP-2 $\alpha$ coated beads or beads coated with GST alone (GST). The gels were silver stained and the proteins retained on GST AP-2 $\alpha$ beads were identified by mass spectrometry. Arrows show the location of Ku80 (up) and Ku70 (down). Molecular masses are indicated on the left and the pH scale on the top of the gels. (b) The presence of AP-2 $\alpha$ and Ku proteins in the eluted fractions was verified by immunoblotting (IB) and compared to a fraction of the input (IN). (c) Co-immunoprecipitation (IP) of AP-2 and Ku proteins by AP-2, Ku70 and Ku80 antibodies or an lg control (Ig CtI). The proteins were revealed by immunoblotting (IB). 
result, [see Additional data file 2] showed a decrease in bound Ku proteins after treatment with low DNase I concentrations. However, the quantity of recovered Ku proteins remained constant when the enzyme concentration was raised, despite the steady decrease in contaminating DNA. This result indicates that the association of Ku to AP-2 is specific.

\section{Ku70/80 depletion induces downregulation of ERBB2 expression}

Figure 2 presents Ku70, Ku80 AP- $2 \alpha$, AP-2 $\gamma$, and p185-erbB2 protein levels in the cytoplasmic and the nuclear fractions of breast cancer (BT-474, SKBR3, ZR-75.1, MCF-7, MDA-MB231), colon cancer (HCT-116) and hepatoma (HepG2) cell lines. In most cells, Ku70 and Ku80 proteins were detected in both the nuclear and the cytoplasmic fraction, with the exception of MCF-7 and HepG2 cell lines where Ku80 was exclusively nuclear. In comparison, AP- $2 \alpha$ and AP- $2 \gamma$ factors were detected only in the nuclear fraction of BT-474, SKBR3 and ZR-75.1 breast cancer cells that overexpress $\mathrm{p} 185^{-\mathrm{erbB} 2}$ protein.

Next, we studied the consequence of Ku70 and Ku80 downregulation on the ERBB2 expression level. For that purpose, $\mathrm{Ku} 70$ and Ku80 were inhibited by the transfection of specific siRNAs in two ERBB2 overexpressing cell lines, BT-474 and SKBR3. In parallel, the cells were transfected with a combination of AP- $2 \alpha$ and AP- $2 \gamma$ (AP-2 $\alpha \gamma$ ) siRNAs, previously shown to downregulate ERBB2 expression [21]. The levels of Ku70, $\mathrm{Ku} 80, \mathrm{AP}-2 \alpha, \mathrm{AP}-2 \gamma$ and $\mathrm{p} 185^{\mathrm{erbB}} 2$ proteins in BT-474 were assessed by western-immunobloting 96 hours after transfection (Figure 3A). Each siRNA inhibited its own target protein. Moreover, Ku70 and Ku80 siRNAs reduced both Ku70 and Ku80 protein levels, in agreement with published data $[35,36]$.

The results of similar experiments on SKBR3 cells are presented in Additional data file 3, Figure A. Ku70 and Ku80 siRNAs reduced p185-erbB2 levels less efficiently in SKBR3 than in BT-474 cells. We have to stress that AP-2 $\alpha \gamma$ siRNAs were also less efficient in SKBR3 than in BT-474 cells.
In order to gain a more precise view of the cooperation between $\mathrm{Ku}$ and $\mathrm{AP}-2$ proteins on ERBB2 gene transcription, the ERBB2 mRNA level was precisely quantified by real-time RT-PCR in $\mathrm{Ku}$ and AP- $2 \alpha \gamma$ siRNA transfected cells. The ERBB2 transcript levels in cells transfected with the siRNAs were compared to the mRNA levels in cells transfected with the Negative (Neg) siRNA [Figure 3B and Figure B in Additional data file 3]. ERBB2 transcript levels were similarly reduced by Ku70 and Ku80 siRNAs in BT474 cells. The AP$2 \alpha \gamma$ siRNAs were more effective, reducing the ERBB2 transcript level by about 50\%. Co-transfection of Ku and AP-2 $\alpha \gamma$ siRNAs did not reduce further the ERBB2 mRNA level in these cells. While the Ku70 siRNA was as effective in SKBR3 as in BT-474 cells, Ku80 and AP-2 ay siRNAs were much less effective. In contrast with BT-474 cells, in SKBR3 cells a combined effect of Ku70 and AP-2 siRNAs was observed.

\section{Ku70/80 regulate ERBB2 promoter transcriptional activity}

To investigate the transcriptional control of ERBB2 gene expression by $\mathrm{Ku}$ and AP-2 proteins, we compared the HCT116 cell line and its derived 70/32 cell line, knocked out for one Ku80 allele [25]. Ku80 protein level in 70/32 cells was decreased by about $40-60 \%$ in comparison with the wild-type HCT116 cells (Figure 4C). The role of the interaction between $\mathrm{Ku}$ and AP-2 on ERBB2 gene expression was studied by transfecting in both cell lines a luciferase- reporter vector containing a wild type or a mutant AP-2 binding site (AP2BS) (Figure $4 A$ ). The cells were cotransfected with $A P-2 \alpha$ and/or Ku80 expression vectors or the corresponding empty vectors. The luciferase activity in the cells transfected with the empty expression vector was considered as equal to one. As previously shown $[13,20], \mathrm{AP}-2 \alpha$ stimulated the activity of the reporter containing the wild type AP2BS (Figure 4B). However, the activation was reduced in 70/32 cells expressing less Ku80. Ku80 expression vector alone had no effect on the promoter activity. However, co-transfection of Ku80 with AP$2 \alpha$ expression vectors induced a significant increase in the promoter activity in both cell lines. Interestingly, transfection of Ku80 restored the activation capacity of AP- $2 \alpha$ in $70 / 32$ cell

Figure 2

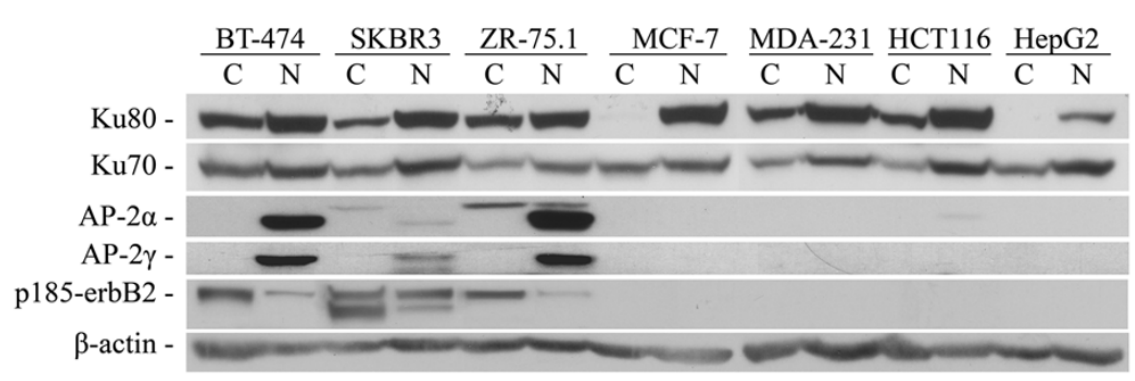

Ku70 and Ku80 protein levels in cytoplasm and nuclei of cancerous cell lines. $60 \mu \mathrm{g}$ of nuclear and cytoplasmic proteins extracted from the cell lines were resolved by $10 \%$ PAGE. The Ku80, Ku70, AP- $2 \alpha$, AP- $2 \gamma$ and $\mathrm{p} 185^{\text {erbB2 }}$ proteins were revealed by immunoblotting. The antibodies are indicated on left. Beta-actin ( $\beta$-actin) protein was used as a loading control. 
Figure 3

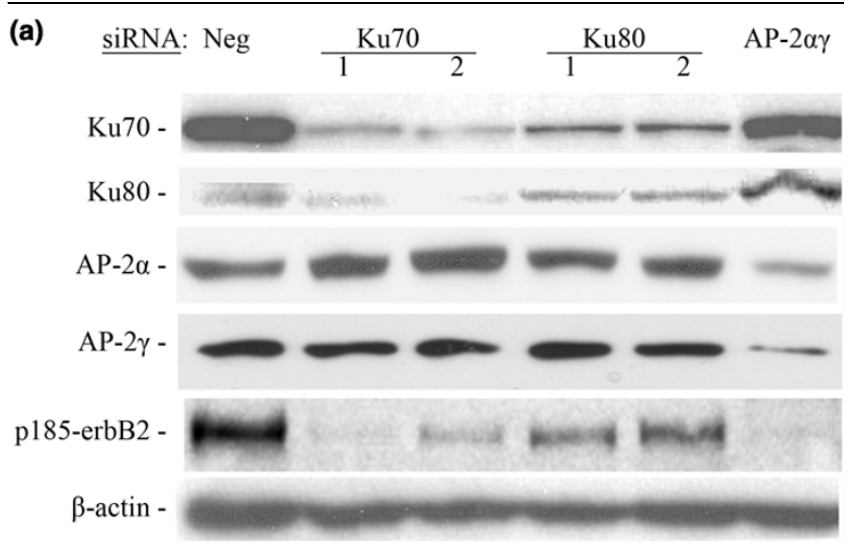

(b)

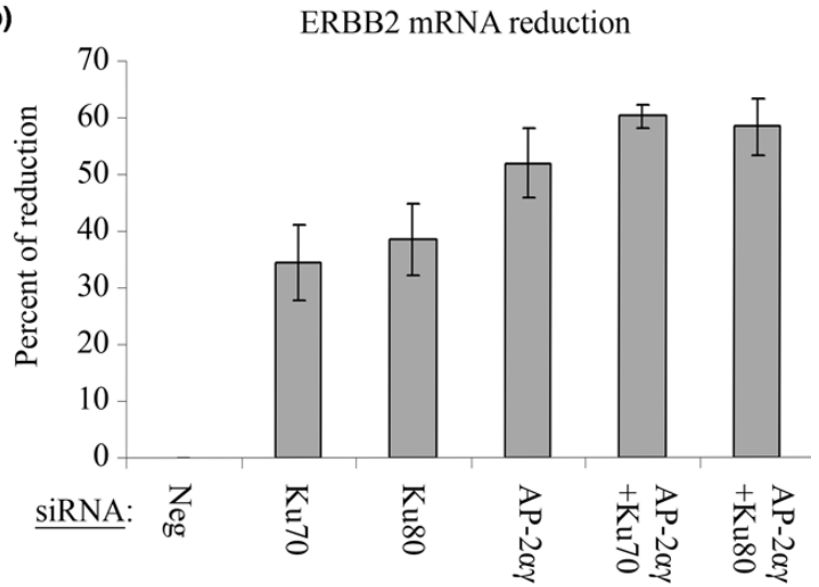

Effect of Ku70/Ku80 and AP-2 downregulation on ERBB2 protein levels in BT-474 cells. (a) Immunoblot showing p185-erbB2, Ku70, Ku80, AP- $2 \alpha$, AP- $2 \gamma$ and beta-actin ( $\beta$-actin) protein levels in cells transfected with the siRNAs indicated above the photograph. (b) Percent reduction of ERBB2 mRNA levels compared to the Neg siRNA condition in cells transfected with the different siRNAs indicated below the figure. ERBB2 mRNA levels were quantified in triplicate by Real-time RT-PCR, and corrected for beta-2-microglobulin mRNA levels.

line. Figure $4 \mathrm{C}$ presents the AP-2 $\alpha$ and Ku80 protein levels in the transfected cells.

To complete this investigation, the reporter vectors activities were measured in BT-474 (Figure 5) and SKBR3 [see Additional data file 4] cells 72 hours after transfection of $\mathrm{Ku}$ and AP-2 siRNAs (Figure 5). The Luciferase activity in cells transfected with the negative siRNA was considered as equal to one in each condition. As expected, downregulation of AP- $2 \alpha$ and AP- $2 \gamma$ inhibited only the activity of the reporter containing the functional AP2BS (p86-AP2BS-Luc). Ku70 or Ku80 siRNAs also inhibited significantly the activity of this reporter vector. Furthermore, when Ku70 or Ku80 siRNAs were cotransfected with the AP- $2 \alpha \gamma$ siRNAs, the activity of p86AP2BS-Luc was further reduced. In contrast, AP-2 $\alpha \gamma$, Ku70
Figure 4

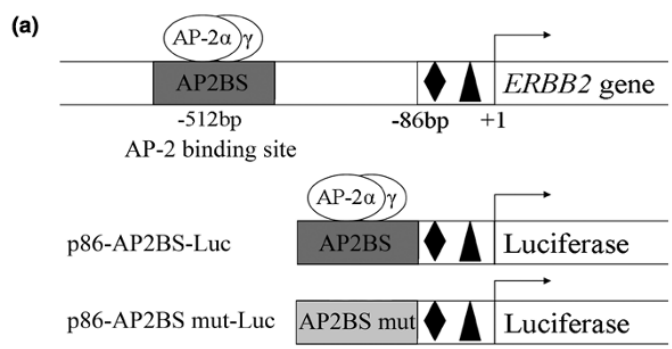

(b)
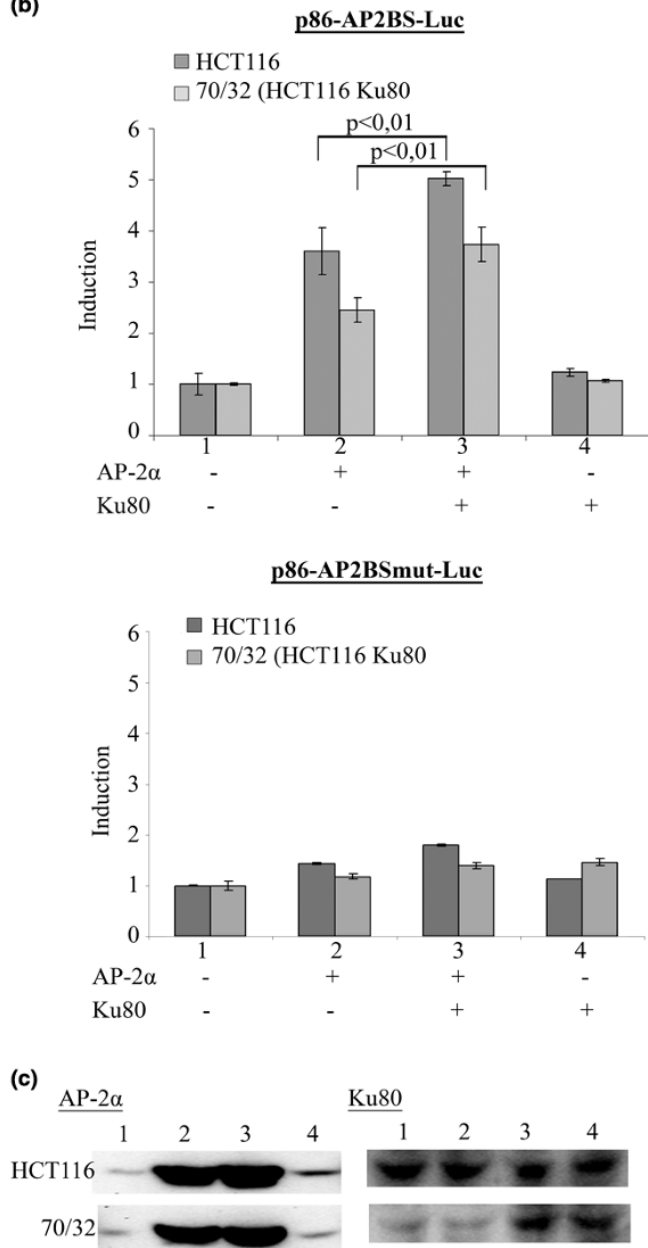

Influence of Ku80 on AP-2 $\alpha$ transcriptional activity on ERBB2 gene promoter. (a) Schematic representation of the reporter vectors used in this study. All the reporter vectors contain 86 bp of the ERBB2 core promoter. The black diamond indicates the CAAT box; the black triangle the TATA box. The sequence of the AP2BS is that of AP-2 binding sites located at -512 bp from the main ERBB2 transcription start site. Mutation of three nucleotides abrogates the binding of the transcription factor to AP2BSmut [25]. (b) Variation of luciferase activity in cells transfected with (+) AP- $\alpha$ expression vector alone or with the Ku80 expression vector, in HCT116 and in 70/32 (HCT116 Ku80+/-) cell lines; compared with the activity in the cells transfected with the corresponding empty expression vectors (-). (c) Western blot showing the levels of AP- $2 \alpha$ and Ku80 proteins in the cells transfected with the corresponding expression vectors. 

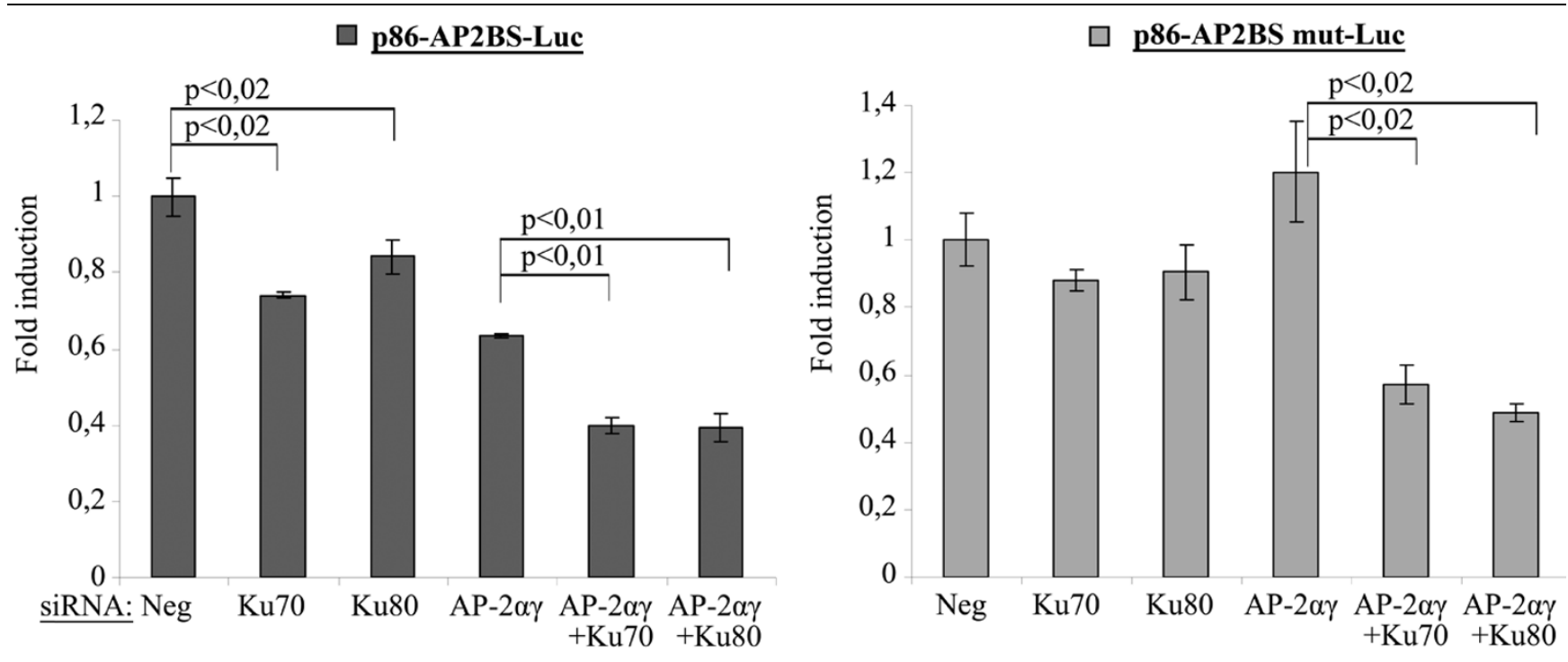

Ku70/80 proteins control ERBB2 promoter activity in BT-474 cell line. Relative luciferase activity in cells transfected with the reporter vectors indicated above the figure, 72 hours after transfection of the siRNAs shown under each bar. The fold induction is the ratio between the luciferase activities in the cells transfected with the siRNA of interest and the activity measured in cells transfected with siRNA Neg. Luciferase activity was normalized to total protein content.

and Ku80 siRNAs did not modify significantly the activity of the vector containing the mutated AP2BS (p86-AP2BS mut-Luc). However, co-transfection of AP-2 $2 \gamma$ with Ku70 or Ku80 significantly reduced the activity of the reporter containing the mutant AP2BS.

Together, these results suggest that $\mathrm{Ku}$ proteins regulate $E R B B 2$ gene expression through the proximal promoter, by an AP-2 dependent mechanism.

\section{Ku70/80 proteins are recruited to the ERBB2 proximal promoter}

Next, we investigated Ku binding to ERBB2 gene promoter by chromatin immunoprecipitation (ChIP). Cross-linked chromatin was extracted from BT-474 (Figure 6A) and SKBR3 cells [Supplemental Data, Figure S.4]. We also extracted chromatin from the same cell type transfected with Ku or AP-2 $\alpha \gamma$ siRNAs. Chromatin fragments were immunoprecipitated with antibodies recognizing AP- $2 \alpha$ and AP-2 $\gamma$ (AP-2), Ku70, Ku80 and RNA Polymerase II (Pol II). The Pol II antibody was used as a positive control for the recruitment of the transcriptional machinery on the ERBB2 promoter [14,20,37]. Three regions of the ERBB2 promoter were amplified (Figure 6B). The -500 bp region contains a high affinity AP2BS [12]. The $-100 \mathrm{bp}$ region contains the CAAT and TATA boxes and corresponds to the $E R B B 2$ core promoter. The -6900 bp sequence was used as an AP2BS negative control [14]. The $\mathrm{Ku}$ binding sequence from the Glucokinase (GCK) gene promoter was used as a positive control for Ku proteins binding [37]. Experiments were repeated three times and the average quantities of DNA were reported to the "No antibody" (NoAb) condition (Figure 6C).

In untreated BT-474 cells, Ku70 antibody immunoprecipitated most efficiently the -500 bp DNA sequence of the ERBB2 gene promoter (Figure $6 \mathrm{C}$, black columns). In agreement with previously published data, AP-2 antibody also immunoprecipitated the same region $[14,20]$. Interestingly, AP-2 antibodies also immunoprecipitated the GCK sequence. The $-500 \mathrm{bp}$, $100 \mathrm{bp}$ and GCK sequences were efficiently recovered from Pol II specific immunoprecipitations. No DNA was recovered in the ChIP experiments using the C-20 Ku80 specific antibody (data not shown). Although the C-20 antibody has been successfully used in our immunoprecipitation experiments (Figure 1C) and published in ChIP data [26], we did not succeed to immunoprecipitate even the GCK control sequence with this antibody. As the $-6900 \mathrm{bp}$ region is devoid of an AP2BS, we consider this signal as background. Compared to BT-474 cells, immunoprecipitation with Ku70 and Polll antibodies of the ERBB2 and GCK promoter regions in SKBR3 was very poor [see Additional data file 5].

AP-2 $\alpha \gamma$ siRNAs reduced significantly the amount of DNA corresponding to the $500 \mathrm{bp}$ region recovered after immunoprecipitation with the AP-2 antibody. Interestingly, AP-2 $\alpha \gamma$ siRNAs drastically reduced all DNA fragments recovered after ChIP using the Ku70 antibody in the BT-474 cell line. The recruitment of the DNA fragment from the GCK promoter after the Ku70 ChIP was also reduced. These siRNAs completely inhibited Pol II recruitment to the ERBB2 and GCK promoters. In the two cell lines, Ku70 siRNA reduced AP-2 recruitment to 
Figure 6

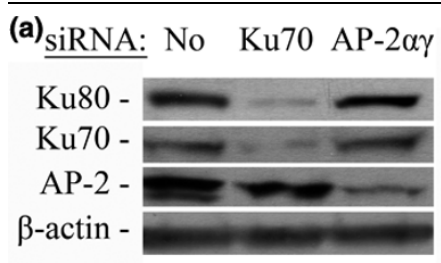

(b)

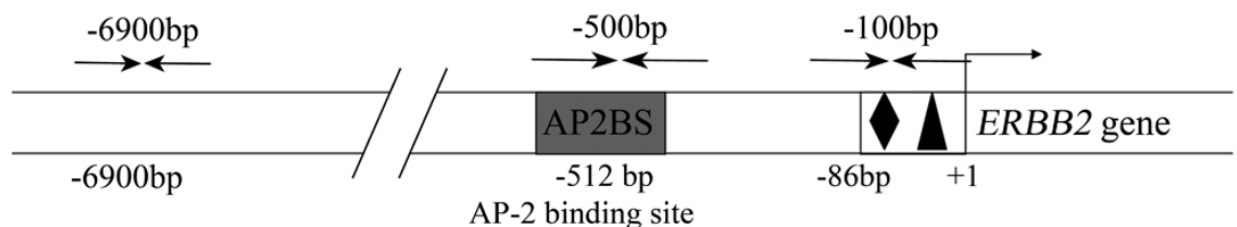

(c)

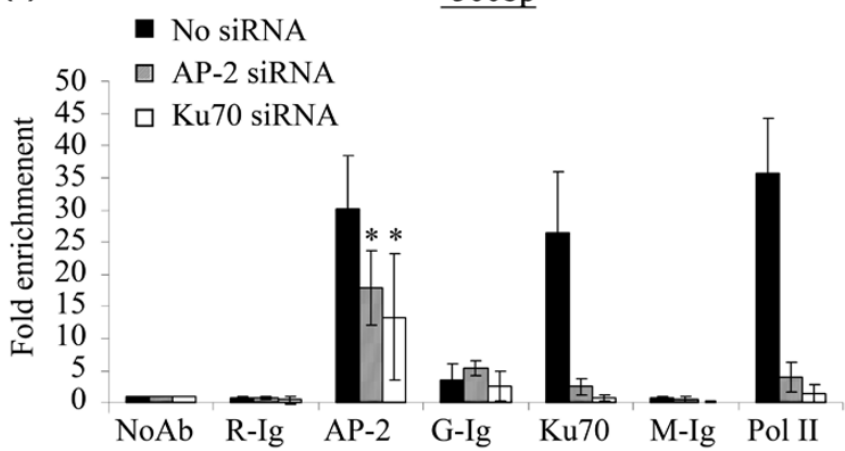

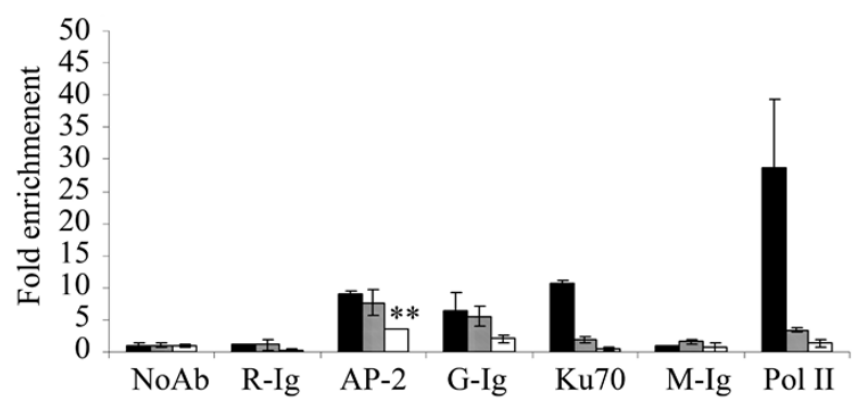

$\underline{\text { GCK }}$
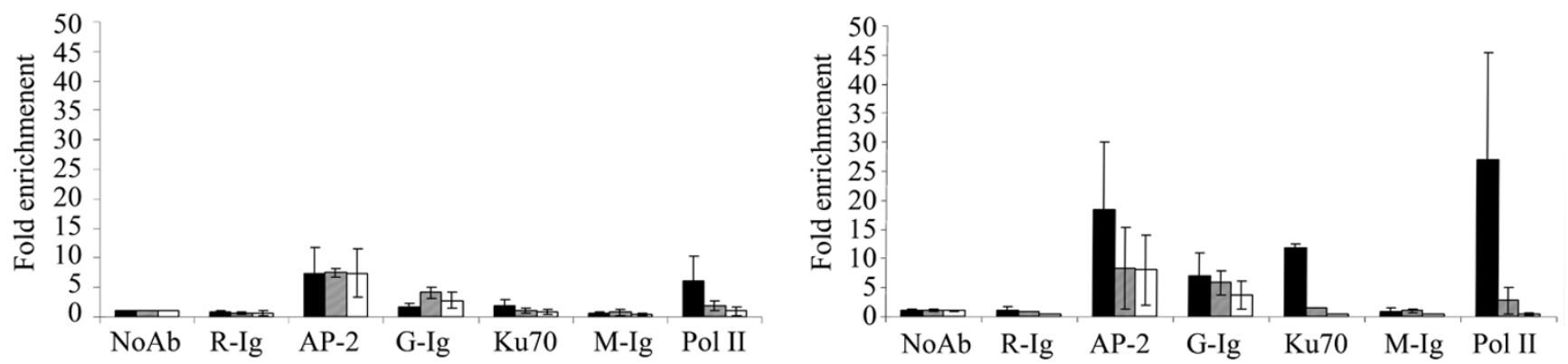

Ku70 is recruited to the ERBB2 promoter and is necessary for the recruitment of AP-2 and of RNA Polymerasell. (a) Chromatin extracted from BT474 cells transfected or not with AP-2 ( $\alpha \gamma)$ or Ku70 siRNAs (above) was shared by sonication to fragments of 100-500 bp. Protein expression was verified by immunoblot. (b) Schematic representation of the ERBB2 promoter illustrating the amplified regions. (c) DNA amplified after chromatin immunoprecipitation with AP-2, Ku70 and RNA Polymerase II (Pol II) specific antibodies. ChIP with control antibodies (Ig) are presented on the left of each experimental point (R: rabbit, G: goat and M: mouse). Graphs show the fold enrichment of the target sequence, compared to the no-antibody (NoAb) condition. The error bars were calculated from the results of three independent ChIP experiments. GCK sequence corresponds to a positive control for Ku recruitment. ${ }^{*}$ and ${ }^{* *}: P$-values $<0,05 \%$ compared to the No siRNA condition.

the ERBB2 and GCK gene promoters. This siRNA also completely inhibited the recruitment of Ku70 and Polll to the promoters we had investigated in the BT-474 cell line.

The ChIP results suggest that both $\mathrm{Ku}$ and AP-2 proteins are recruited on the ERBB2 proximal promoter in BT-474 cells. Moreover, the binding of both factors is necessary for the recruitment of transcription machinery on the ERBB2 gene promoter in BT-474 and SKBR3 cell lines.

\section{Discussion}

The aim of this study was to identify novel proteins interacting with and contributing to AP-2 transcription factor activity. Using GST-pull-down coupled with two-dimensional gel electrophoresis and mass spectrometry, Ku70 and Ku80 proteins were identified as AP-2 $\alpha$ interactors. AP-2/Ku interaction was confirmed by co-immunoprecipitation. We showed that downregulation of Ku proteins by siRNAs induced a strong reduction in ERBB2 mRNA and protein levels in BT-474 and SKBR3 cells. These siRNAs also inhibited the activity of reporter vectors containing the ERBB2 proximal promoter. ChIP experiments revealed that Ku70 proteins were recruited 
to the $E R B B 2$ promoter. Interestingly, the inhibition of AP- $2 \alpha$ and AP- $2 \gamma$ expression by siRNA strongly reduced Ku70 recruitment to the $E R B B 2$ promoter. Ku70 siRNA reduced by half the recruitment of AP-2 factors to the -500 bp region of the $E R B B 2$ promoter containing a high affinity AP2BS. More importantly, Ku70 siRNA downregulated Polll recruitment to the ERBB2 promoter. These results show that the Ku proteins are involved in $E R B B 2$ gene expression regulation by AP-2 in breast cancer cells.

In addition to their role in the repair of DNA double strand breaks, Ku proteins have been shown to control other important cellular processes such as transcription and apoptosis. $\mathrm{Ku}$ proteins modulate transcription by several mechanisms and these properties seem to be gene and cell specific. For example, DNA binding of the Ku70/Ku80 heterodimer is responsible for the downregulation of glycoprotein glycophorin $\mathrm{B}$ in non erythroid cells [38]. In contrast, Ku binding to apolipoprotein C-IV promoter stimulates the expression of the gene [39]. Another study showed that interleukins -13/-4 induced expression of the lipooxygenase-1 gene is mediated by the binding of $\mathrm{Ku}$ dimmers to the promoter [40]. C-jun expression is also stimulated by Ku80 and possibly by Ku70 binding to gene promoter [41]. Ku proteins can also influence transcription by interaction with transcription factors or cofactors. So, Ku binding inhibits ESE1, an Ets family transcription factor, from binding to DNA and thus its transcriptional activity [42]. Ku proteins might also be involved in elongation [37] and transcription reinitialization [43].

Ku proteins were also shown to modulate gene expression by binding to PARP-1 and to YY1, which were shown to interact with and influence AP-2 transcription factor activity. Indeed, PARP-1 is necessary to preserve the transcriptional activity of overexpressed AP-2 transcription factors [18]. The Ku - PARP interaction has opposing effects on transcription. Ku proteins inhibit the transcriptional activity of $\beta$-catenin-TCF4 complex by interfering with PARP-1 binding [44]. In contrast, the Ku70/ Ku80 dimmer - PARP-1 complex stimulates the expression of the S10019 gene [45]. Ku - YY1 interaction inhibits $\alpha$ myosin heavy-chain gene expression in the heart [46], contrary to the consequence of $Y Y 1$ interaction with AP-2 on ERBB2 gene expression [20].

\section{Conclusions}

In summary, the present study contributes to a better understanding of the regulation of ERBB2 gene expression in breast cancer cells, by demonstrating the implication of Ku proteins together with AP-2 transcription factors in the expression of the oncogene. Our results implicate Ku proteins in breast cancer by contributing to ERBB2 gene overexpression and thus the accumulation of excessive amounts of the receptor. Understanding the mechanisms of ERBB2 gene deregulation might help in the development of drugs that target further key elements responsible for this important deregulation. Among these, inhibiting Ku activity might be evaluated as an adjuvant for ERBB2 targeted therapy in breast cancer. However, more work is needed in models resembling the in vivo situation to confirm the implication of Ku proteins in the accumulation of excessive amounts of ERBB2 protein in cancerous cells.

\section{Competing interests}

The authors declare that they have no financial competing interests.

\section{Authors' contributions}

GN carried out all the studies and drafted the manuscript. JCP and $B K$ helped in the studies and interpretation of the results. $\mathrm{BE}, \mathrm{WZ}$ and EDP participated in the study design, revised the manuscript and provided important intellectual support. RW conceived of the study, participated in its design, coordination and interpretation of the results and finalized the manuscript. All authors read and approved the final manuscript.

\section{Additional files}

The following Additional files are available online:

\section{Additional data file 1}

A PDF file showing Mass Spectometry identification of the $\mathrm{Ku}$ proteins interacting with AP- $2 \alpha$.

See http://www.biomedcentral.com/content/ supplementary/bcr2450-S1.pdf

\section{Additional file 2}

A PDF file showing that $\mathrm{Ku}$ and AP-2 protein interaction is specific.

See http://www.biomedcentral.com/content/ supplementary/bcr2450-S2.pdf

\section{Additional file 3}

A PDF file showing the effect of Ku70/Ku80 and AP-2 deregulation on ERBB2 $\mathrm{mRNA}$ and protein expression in SKBR3 cells.

See http://www.biomedcentral.com/content/ supplementary/bcr2450-S3.pdf

\section{Additional file 4}

A PDF file showing that Ku70/80 proteins control ERBB2 promoter activity in SKBR3 cell line.

See http://www.biomedcentral.com/content/ supplementary/bcr2450-S4.pdf

\section{Additional file 5}

A PDF file showing that Ku proteins are necessary for the recruitment of AP-2 on the proximal ERBB2 gene promoter in SKBR3 cell line.

See http://www.biomedcentral.com/content/ supplementary/bcr2450-S5.pdf 


\section{Acknowledgements}

We thank Dr Kannan, Dr Hendrickson and Dr. Chen for their gifts. We thank Dr. Gabriel Mazzucchelli for his help in Mass Spectrometry analyses. This work was supported by grants from Televie (Belgium), Anticancer Centre attached to the University of Liege (Belgium).

GN and JCP are recipients of Televie grants from the FNRS; BK is research fellow of the FNRS; BE and WZ are researchers financially supported by Région Wallonne, contract BA4 grant 114915, contract EPH331030000092-430001, ЕРН331030000022-130033, Fonds Social Européen contract W2002134, W1000346, contract 14531, iPCRq; EDP is professor at the University of Liege, director of the Mass Spectrometry Laboratory; RW is research director of the FNRS. Support is via FRSM Grant no. 3.4.542.04 and from the Centre anticancéreux at the University of Liège.

\section{References}

1. Ferlay J, Autier P, Boniol M, Heanue M, Colombet M, Boyle P: Estimates of the cancer incidence and mortality in Europe in 2006. Ann Oncol 2007, 18:581-592.

2. Sorlie T, Perou CM, Tibshirani R, Aas T, Geisler S, Johnsen H, Hastie T, Eisen MB, Riin M van de, Jeffrey SS, Thorsen T, Quist $H$, Matese JC, Brown PO, Botstein D, Eystein LP, Borresen-Dale AL: Gene expression patterns of breast carcinomas distinguish tumor subclasses with clinical implications. Proc Natl Acad Sci USA 2001, 98:10869-10874.

3. Sorlie T, Perou CM, Fan C, Geisler S, Aas T, Nobel A, Anker G, Akslen LA, Botstein D, Borresen-Dale AL, Lonning PE: Gene expression profiles do not consistently predict the clinical treatment response in locally advanced breast cancer. Mol Cancer Ther 2006, 5:2914-2918.

4. Ferretti G, Felici A, Papaldo P, Fabi A, Cognetti F: HER2/neu role in breast cancer: from a prognostic foe to a predictive friend. Curr Opin Obstet Gynecol 2007, 19:56-62.

5. Hynes NE, Lane HA: ERBB receptors and cancer: the complexity of targeted inhibitors. Nat Rev Cancer 2005, 5:341-354.

6. Ross JS, Fletcher JA: The HER-2/neu Oncogene in Breast Cancer: Prognostic Factor, Predictive Factor, and Target for Therapy. Oncologist 1998, 3:237-252.

7. Slamon DJ, Clark GM, Wong SG, Levin WJ, Ullrich A, McGuire WL: Human breast cancer: correlation of relapse and survival with amplification of the HER-2/neu oncogene. Science 1987, 235:177-182.

8. Pasleau F, Grooteclaes M, Gol-Winkler R: Expression of the cerbB2 gene in the BT474 human mammary tumor cell line: measurement of c-erbB2 mRNA half-life. Oncogene 1993, 8:849-854.

9. Scott GK, Chang CH, Erny KM, Xu F, Fredericks WJ, Rauscher FJ III, Thor AD, Benz CC: Ets regulation of the erbB2 promoter. Oncogene 2000, 19:6490-6502.

10. Wu J, Lee C, Yokom D, Jiang H, Cheang MC, Yorida E, Turbin D, Berquin IM, Mertens PR, Iftner T, Gilks CB, Dunn SE: Disruption of the Y-box binding protein-1 results in suppression of the epidermal growth factor receptor and HER-2. Cancer Res 2006, 66:4872-4879.

11. Bosher JM, Williams T, Hurst HC: The developmentally regulated transcription factor AP-2 is involved in c-erbB-2 overexpression in human mammary carcinoma. Proc Natl Acad Sci USA 1995, 92:744-747.

12. Grooteclaes M, Vernimmen D, Plaza S, Pasleau F, Hodzic D, Winkler-Gol R: A new cis element is involved in the HER2 gene overexpression in human breast cancer cells. Cancer Res 1999, 59:2527-2531.

13. Vernimmen D, Begon D, Salvador C, Gofflot S, Grooteclaes M, Winkler R: Identification of HTF (HER2 transcription factor) as an AP-2 (activator protein-2) transcription factor and contribution of the HTF binding site to ERBB2 gene overexpression. Biochem J 2003, 370:323-329.

14. Delacroix L, Begon D, Chatel G, Jackers $P$, Winkler R: Distal ERBB2 promoter fragment displays specific transcriptional and nuclear binding activities in ERBB2 overexpressing breast cancer cells. DNA Cell Biol 2005, 24:582-594.
15. Bosher JM, Totty NF, Hsuan JJ, Williams T, Hurst HC: A family of AP-2 proteins regulates c-erbB-2 expression in mammary carcinoma. Oncogene 1996, 13:1701-1707.

16. Pellikainen JM, Kosma VM: Activator protein-2 in carcinogenesis with a special reference to breast cancer--a mini review. Int J Cancer 2007, 120:2061-2067.

17. Kannan P, Buettner R, Chiao PJ, Yim SO, Sarkiss M, Tainsky MA: $\mathrm{N}$-ras oncogene causes AP-2 transcriptional self-interference, which leads to transformation. Genes Dev 1994, 8:1258-1269.

18. Kannan $P, Y u$ Y, Wankhade S, Tainsky MA: PolyADP-ribose polymerase is a coactivator for AP-2-mediated transcriptional activation. Nucleic Acids Res 1999, 27:866-874.

19. Braganca J, Swingler T, Marques FI, Jones T, Eloranta JJ, Hurst HC, Shioda T, Bhattacharya S: Human CREB-binding protein/ p300-interacting transactivator with ED-rich tail (CITED) 4, a new member of the CITED family, functions as a co-activator for transcription factor AP-2. J Biol Chem 2002, 277:8559-8565

20. Begon DY, Delacroix L, Vernimmen D, Jackers $P$, Winkler R: Yin Yang 1 cooperates with activator protein 2 to stimulate ERBB2 gene expression in mammary cancer cells. J Biol Chem 2005, 280:24428-24434

21. Allouche A, Nolens G, Tancredi A, Delacroix L, Mardaga J, Fridman V, Winkler R, Boniver J, Delvenne P, Begon DY: The combined immunodetection of AP-2alpha and YY1 transcription factors is associated with ERBB2 gene overexpression in primary breast tumors. Breast Cancer Res 2008, 10:R9.

22. Li M, Wang $Y$, Hung MC, Kannan P: Inefficient proteasomaldegradation pathway stabilizes AP-2alpha and activates HER2/neu gene in breast cancer. Int J Cancer 2006, 118:802-811.

23. Featherstone $\mathrm{C}$, Jackson SP: Ku, a DNA repair protein with multiple cellular functions? Mutat Res 1999, 434:3-15.

24. Gullo C, Au M, Feng G, Teoh G: The biology of Ku and its potential oncogenic role in cancer. Biochim Biophys Acta 2006 , 1765:223-234.

25. $\mathrm{Li} \mathrm{G}$, Nelsen $\mathrm{C}$, Hendrickson EA: Ku86 is essential in human somatic cells. Proc Natl Acad Sci USA 2002, 99:832-837.

26. Matheos D, Novac O, Price GB, Zannis-Hadjopoulos M: Analysis of the DNA replication competence of the xrs-5 mutant cells defective in Ku86. J Cell Sci 2003, 116:111-124.

27. Zhang S, Schlott B, Gorlach M, Grosse F: DNA-dependent protein kinase (DNA-PK) phosphorylates nuclear DNA helicase II/ RNA helicase $A$ and hnRNP proteins in an RNA-dependent manner. Nucleic Acids Res 2004, 32:1-10.

28. Buettner R, Kannan P, Imhof A, Bauer R, Yim SO, Glockshuber R, Van Dyke MW, Tainsky MA: An alternatively spliced mRNA from the AP-2 gene encodes a negative regulator of transcriptional activation by AP-2. Mol Cell Biol 1993, 13:4174-4185.

29. Gaubatz S, Imhof A, Dosch R, Werner O, Mitchell P, Buettner R, Eilers M: Transcriptional activation by Myc is under negative control by the transcription factor AP-2. EMBO J 1995, 14:1508-1519.

30. Ishiguro A, Ideta M, Mikoshiba K, Chen DJ, Aruga J: ZIC2-dependent transcriptional regulation is mediated by DNA-dependent protein kinase, poly(ADP-ribose) polymerase, and RNA helicase A. J Biol Chem 2007, 282:9983-9995.

31. Francis F, Gerkens P Harmel N, Mazzucchelli G, De Pauw PE Haubruge E: Proteomics in Myzus persicae: effect of aphid host plant switch. Insect Biochem Mol Bio/ 2006, 36:219-227.

32. Chaplet M, Detry C, Deroanne C, Fisher LW, Castronovo V, Bellahcene A: Zoledronic acid up-regulates bone sialoprotein expression in osteoblastic cells through Rho GTPase inhibition. Biochem J 2004, 384:591-598.

33. Dahl JA, Collas P: A rapid micro chromatin immunoprecipitation assay (microChIP). Nat Protoc 2008, 3:1032-1045.

34. Nguyen TN, Goodrich JA: Protein-protein interaction assays: eliminating false positive interactions. Nat Methods 2006 , 3:135-139.

35. Bertolini LR, Bertolini M, Anderson GB, Maga EA, Madden KR, Murray JD: Transient depletion of Ku70 and Xrcc4 by RNAi as a means to manipulate the non-homologous end-joining pathway. J Biotechno/ 2007, 128:246-257.

36. Mayeur GL, Kung WJ, Martinez A, Izumiya C, Chen DJ, Kung $\mathrm{HJ}$ : $\mathrm{Ku}$ is a novel transcriptional recycling coactivator of the androgen receptor in prostate cancer cells. J Biol Chem 2005, 280:10827-10833. 
37. Mo X, Dynan WS: Subnuclear localization of Ku protein: functional association with RNA polymerase II elongation sites. Mol Cell Biol 2002, 22:8088-8099.

38. Camara-Clayette V, Rahuel C, Bertrand O, Cartron JP: The E-box of the human glycophorin B promoter is involved in the erythroid-specific expression of the GPB gene. Biochem Biophys Res Commun 1999, 265:170-176.

39. Kim E, Li K, Lieu C, Tong S, Kawai S, Fukutomi T, Zhou Y, Wands $\mathrm{J}, \mathrm{Li} \mathrm{J}$ : Expression of apolipoprotein C-IV is regulated by Ku antigen/peroxisome proliferator-activated receptor gamma complex and correlates with liver steatosis. J Hepatol 2008, 49:787-798.

40. Kelavkar UP, Wang S, Badr KF: Ku autoantigen (DNA helicase) is required for interleukins-13/-4-induction of 15-lipoxygenase-1 gene expression in human epithelial cells. Genes Immun 2000, 1:237-250.

41. Jiang D, Zhou Y, Moxley RA, Jarrett HW: Purification and identification of positive regulators binding to a novel element in the c-Jun promoter. Biochemistry 2008, 47:9318-9334.

42. Wang H, Fang R, Cho JY, Libermann TA, Oettgen P: Positive and negative modulation of the transcriptional activity of the ETS factor ESE-1 through interaction with p300, CREB-binding protein, and Ku 70/86. J Biol Chem 2004, 279:25241-25250.

43. Woodard RL, Lee KJ, Huang J, Dynan WS: Distinct roles for Ku protein in transcriptional reinitiation and DNA repair. J Biol Chem 2001, 276:15423-15433.

44. Idogawa M, Masutani M, Shitashige M, Honda K, Tokino T, Shinomura Y, Imai K, Hirohashi S, Yamada T: Ku70 and poly(ADPribose) polymerase-1 competitively regulate beta-catenin and T-cell factor-4-mediated gene transactivation: possible linkage of DNA damage recognition and Wnt signaling. Cancer Res 2007, 67:911-918.

45. Grote J, Konig S, Ackermann D, Sopalla C, Benedyk M, Los M, Kerkhoff C: Identification of poly(ADP-ribose)polymerase-1 and $\mathrm{Ku} 70 / \mathrm{Ku} 80$ as transcriptional regulators of S100A9 gene expression. BMC Mol Biol 2006, 7:48.

46. Sucharov CC, Helmke SM, Langer SJ, Perryman MB, Bristow M, Leinwand L: The Ku protein complex interacts with YY1, is upregulated in human heart failure, and represses alpha myosin heavy-chain gene expression. Mol Cell Biol 2004, 24:8705-8715. 